\title{
Secret Key Generation for a Pairwise Independent Network Model
}

\author{
Sirin Nitinawarat, Student Member, IEEE, Chunxuan Ye, Senior Member, IEEE, Alexander Barg, Fellow, IEEE, \\ Prakash Narayan, Fellow, IEEE, and Alex Reznik, Member, IEEE
}

\begin{abstract}
We consider secret key generation for a "pairwise independent network" model in which every pair of terminals observes correlated sources that are independent of sources observed by all other pairs of terminals. The terminals are then allowed to communicate publicly with all such communication being observed by all the terminals. The objective is to generate a secret key shared by a given subset of terminals at the largest rate possible, with the cooperation of any remaining terminals. Secrecy is required from an eavesdropper that has access to the public interterminal communication. A (single-letter) formula for secret key capacity brings out a natural connection between the problem of secret key generation and a combinatorial problem of maximal packing of Steiner trees in an associated multigraph. An explicit algorithm is proposed for secret key generation based on a maximal packing of Steiner trees in a multigraph; the corresponding maximum rate of Steiner tree packing is thus a lower bound for the secret key capacity. When only two of the terminals or when all the terminals seek to share a secret key, the mentioned algorithm achieves secret key capacity in which case the bound is tight.
\end{abstract}

Index Terms-PIN model, private key, public communication, secret key capacity, security index, spanning tree packing, Steiner tree packing, wiretap secret key.

\section{INTRODUCTION}

$\mathbf{S}$ UPPOSE that terminals $1, \ldots, m$ observe distinct but correlated signals with the feature that every pair of terminals observes a corresponding pair of correlated signals that are independent of all other pairs of signals. Following these observations, all the terminals can communicate interactively over a public noiseless channel of unlimited capacity, with all such communication being observed by all the terminals. The goal is to generate a secret key (SK), i.e., secret common randomness, for a given subset $A$ of the terminals in $\mathcal{M}=\{1, \ldots, m\}$ at the largest rate possible, with secrecy being required from an eavesdropper that observes the public interterminal communication.

Manuscript received November 06, 2009; revised July 20, 2010. Date of current version November 19, 2010. The work of S. Nitinawarat and P. Narayan was supported by the National Science Foundation by Grants CCF0515124, CCF0635271, CCF0830697, and InterDigital. The work of A. Barg was supported by the National Science Foundation by Grants CCF0515124, CCF0830699, CCF0916919, DMS0807411, and InterDigital. The material in this paper was presented at the IEEE International Symposium on Information Theory, Nice, France, June 2007, and at the IEEE International Symposium on Information Theory, Toronto, ON, Canada, July 2008.

S. Nitinawarat, A. Barg, and P. Narayan are with the Department of Electrical and Computer Engineering and the Institute for Systems Research, University of Maryland, College Park, MD 20742 USA (e-mail: nitinawa@umd.edu; abarg@umd.edu; prakash@umd.edu).

C. Ye and A. Reznik are with InterDigital, King of Prussia, PA 19406 USA (e-mail: Chunxuan.Ye@interdigital.com; Alex.Reznik@interdigital.com).

Communicated by E. Erkip, Associate Editor for Shannon Theory.

Digital Object Identifier 10.1109/TIT.2010.2081210
All the terminals in $\mathcal{M}$ cooperate in generating the SK for the secrecy-seeking set $A$.

This model for SK generation, called a "pairwise independent network" model, was introduced in [23] (see also [22]). Abbreviated hereafter as the PIN model, it is motivated by practical aspects of a wireless communication network in which terminals communicate on the same frequency. In a typical multipath environment, the wireless channel between each pair of terminals produces a random mapping between the transmitted and received signals which is time-varying and location-specific. For a fixed time and location, this mapping is reciprocal, i.e., effectively the same in both directions. Also, the mapping decorrelates over different time-coherence intervals as well as over distances of the order of a few wavelengths.

The PIN model is, in fact, a special case of a general multiterminal "source model" for secrecy generation studied by Csiszár and Narayan [4]. The latter followed leading investigations by Maurer [13], [14] and Ahlswede and Csiszár [1] of SK generation by two terminals from their correlated observations complemented by public communication.

A single-letter characterization of secret key capacity-the largest rate at which secrecy can be generated-for the terminals in an arbitrary subset $A$ of $\mathcal{M}$ was provided in [4]. A particularization of this (general) SK capacity formula to our PIN model displays the special feature that it can be expressed in terms of a linear combination of mutual information terms that involve only mutually independent pairs of "reciprocal" random variables (rvs). Each such mutual information term represents the maximum rate of an SK that can be generated solely by a corresponding pair of terminals from only their own observed signals using public communication [13], [14], [1]. This observation leads to the following question that is our main motivation: Can an SK of optimum rate for the terminals in A be generated by propagating mutually independent and rate-optimal SKs for pairs of terminals in $\mathcal{M}$ ?

An examination of this question brings out points of contact between SK generation for a PIN model and a combinatorial problem of tree packing in a multigraph. We propose an explicit algorithm for propagating pairwise SKs for pairs of terminals in $\mathcal{M}$ to form a groupwide SK for the terminals in $A$. This algorithm is based on a maximal packing of Steiner trees (for $A$ ) in a multigraph associated with the PIN model. Thus, the maximum rate of Steiner tree packing in this multigraph is always a lower bound for SK capacity. This bound is shown to be tight when the secrecy-seeking set $A$ contains only two terminals or when it consists of all the terminals. In these situations, our algorithm is capacity-achieving. It is of independent interest to note that given a combinatorial problem of determining the max- 
imum rate of Steiner tree packing for $A$ in a multigraph, the SK capacity of an associated PIN model provides, in reciprocity, an upper bound for the mentioned rate, which is tight for the case $|A|=2$ as well as for the spanning tree case $A=\mathcal{M}$.

In the study of secrecy generation for a multiterminal source model, the notions of wiretap SK [13], [14], [1], [4] and private key [4] also have been proposed. The former notion corresponds to the eavesdropper having additional access to a terminal not in the secrecy-seeking set $A$ and from which too the key must be concealed; this "wiretapped" terminal does not cooperate in secrecy generation. A single-letter characterization of the corresponding capacity remains unresolved in general but for partial results and bounds (cf., e.g., [1], [14], [19], [4], [9], [10], [5]). The notion of a private key is less restrictive, with the wiretapped terminal being allowed to cooperate; the corresponding capacity is known [4]. We argue in Section IV below that for a PIN model these two notions correspond to SK generation for a reduced PIN model, thereby justifying our sole focus on SK capacity.

Basic concepts and definitions are presented in Section II. Section III contains statements of our results and proofs; specifically, the SK capacity for the PIN model is given in Section III-A, the connection of SK capacity with Steiner tree packing is treated in Section III-B, and with spanning tree packing in Section III-C. Concluding remarks and pointers to a sequel paper are contained in Section IV.

\section{PRELIMINARIES}

We shall be concerned throughout with a PIN model, which is a special case of a general multiterminal "source model" for secrecy generation with public communication (see [14], [1], [4], [5]). Suppose that terminals $1, \ldots, m, m \geq 2$, observe $n$ independent and identically distributed (i.i.d.) repetitions of the rvs $\tilde{X}_{1}, \ldots, \tilde{X}_{m}$, denoted by $\tilde{X}_{1}^{n}, \ldots, \tilde{X}_{m}^{n}$, where $\tilde{X}_{i}^{n}=$ $\left(\tilde{X}_{i, 1}, \ldots, \tilde{X}_{i, n}\right), i \in \mathcal{M}=\{1, \ldots, m\}$. Each rv $\tilde{X}_{i}, i \in \mathcal{M}$, is of the form $\tilde{X}_{i}=\left(X_{i j}, j \in \mathcal{M} \backslash\{i\}\right)$ with $m-1$ components, and the "reciprocal pairs" of rvs $\left\{\left(X_{i j}, X_{j i}\right), 1 \leq i<j \leq m\right\}$ are mutually independent. See Fig. 1 . Thus, every pair of terminals in $\mathcal{M}$ is associated with a corresponding pair of rvs that are independent of pairs of rvs associated with all the other pairs of terminals. All the rvs are assumed to take their values in finite sets. Following their observation of the random sequences as above, the terminals in $\mathcal{M}$ are allowed to communicate among themselves over a public noiseless channel of unlimited capacity; all such communication, which may be interactive and conducted in multiple rounds, is observed by all the terminals. A communication from a terminal, in general, can be any function of its observed sequence as well as all previous public communication. The public communication of all the terminals will be denoted collectively by $\mathbf{F}=\mathbf{F}^{(n)}$.

The overall goal is to generate shared secret common randomness for a given set $A \subseteq \mathcal{M}$ of terminals at the largest rate possible, with the remaining terminals (if any) cooperating in secrecy generation. The resulting secret key must be shared by every terminal in $A$; but it need not be accessible to the terminals not in $A$ and nor does it need to be concealed from them. It must, of course, be kept secret from the eavesdropper that has

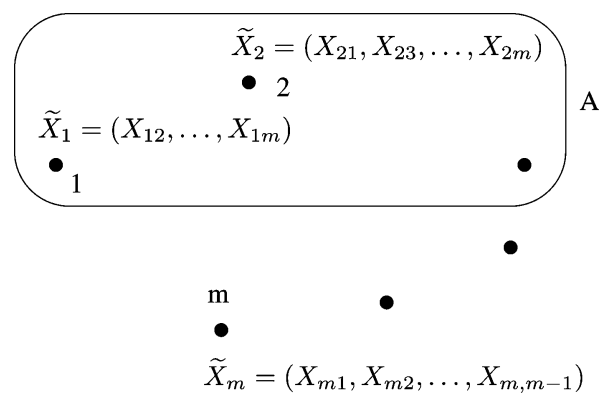

Fig. 1. The PIN Model.

access to the public interterminal communication $\mathbf{F}$, but is otherwise passive, i.e., unable to tamper with this communication.

The following basic concepts and definitions are from [4], [5]. Given $\epsilon>0$, for rvs $U, V$, we say that $U$ is $\epsilon$-recoverable from $V$ if $\operatorname{Pr}\{U \neq f(V)\} \leq \epsilon$ for some function $f(V)$ of $V$. With the rvs $K$ and $\mathbf{F}$ representing a secret key and the eavesdropper's knowledge, respectively, information theoretic secrecy entails that the security index ${ }^{1}$

$$
s(K ; \mathbf{F})=\log |\mathcal{K}|-H(K \mid \mathbf{F})
$$

be required to be small, where $\mathcal{K}$ is the range of $K$ and $|$. denotes cardinality. This requirement simultaneously renders $K$ to be nearly uniformly distributed and nearly independent of $\mathbf{F}$.

Definition 1: Given any set $A \subseteq \mathcal{M}$ of size $|A| \geq 2$, a rv $K$ constitutes an $\epsilon$-secret key ( $\epsilon$-SK) for the set of terminals $A$, achievable with communication $\mathbf{F}$, if $K$ is $\epsilon$-recoverable from $\left(\tilde{X}_{i}^{n}, \mathbf{F}\right)$ for each $i \in A$ and, in addition, it satisfies the secrecy condition

$$
s(K ; \mathbf{F}) \leq \epsilon .
$$

The condition (1) corresponds to the concept of "strong" secrecy in which $\epsilon=\epsilon_{n}=o_{n}(1)$ [15], [4], [5], as distinct from the earlier "weak" secrecy concept which requires only that $\epsilon_{n}=o(n)$ [14], [1].

Definition 2: A number $R$ is an achievable $\mathrm{SK}$ rate for a set of terminals $A \subseteq \mathcal{M}$ if there exist $\epsilon_{n}$-SKs $K^{(n)}$ for $A$, achievable with communication $\mathbf{F}$, such that

$$
\epsilon_{n} \rightarrow 0 \text { and } \frac{1}{n} \log \left|\mathcal{K}^{(n)}\right| \rightarrow R \text { as } n \rightarrow \infty .
$$

The largest achievable SK rate for $A$ is the SK capacity $C(A)$.

Thus, by definition, the SK capacity for $A$ is the largest rate of a rv that is recoverable at each terminal in $A$ from the information available to it, and is nearly uniformly distributed and effectively concealed from an eavesdropper with access to the public interterminal communication; it need not be concealed from the terminals in $A^{c}=\mathcal{M} \backslash A$ that cooperate in secrecy generation.

A single-letter characterization of the SK capacity $C(A)$, $A \subseteq \mathcal{M}$, for a general multiterminal source model, of which

\footnotetext{
${ }^{1}$ All logarithms are to the base 2 .
} 
the PIN model is a special case, is provided in [4]. An upper bound for $C(A)$ in terms of (Kullback-Leibler) divergence is also given therein and shown to be tight in special cases. These results play material roles below.

\section{RESULTS}

Our main results are the following. First, we obtain, upon particularizing the results of [4], a (single-letter) expression for $C(A)$ for a PIN model, in terms of a linear combination of mutual information terms that involve only pairs of "reciprocal" rvs $\left\{\left(X_{i j}, X_{j i}\right), 1 \leq i \neq j \leq m\right\}$. Second, stemming from this observation, a connection is drawn between SK generation for the PIN model and the combinatorial problem of maximal packing of Steiner trees in an associated multigraph. Specifically, we show that the maximum rate of Steiner tree packing in the multigraph is always a lower bound for SK capacity. Third, for the case $|A|=2$ (when the Steiner tree becomes a path connecting the two vertices in $A$ ) and for the case $A=\mathcal{M}$ (when the Steiner tree becomes a spanning tree), the previous lower bound is shown to be tight. This is done by means of an explicit algorithm, based on maximal path packing and maximal spanning tree packing, respectively, that forms an SK out of independent SKs for pairs of terminals. In fact, the maximum rate of the SK thereby generated equals the previously known upper bound for SK capacity [4] mentioned above.

\section{A. SK Capacity}

We first give the SK capacity $C(A)$ for the PIN model. For $A \subseteq \mathcal{M}$, let

$$
\mathcal{B}(A)=\{B \subset \mathcal{M}: B \neq \emptyset, B \nsupseteq A\}
$$

and $\mathcal{B}_{i}(A)$ be its subset consisting of those $B \in \mathcal{B}(A)$ that contain $i, i \in \mathcal{M}$. Let $\Lambda(A)$ be the set of all collections $\lambda=$ $\left\{\lambda_{B}: B \in \mathcal{B}(A)\right\}$ of weights $0 \leq \lambda_{B} \leq 1$, satisfying

$$
\sum_{B \in \mathcal{B}_{i}(A)} \lambda_{B}=1 \text { for all } i \in \mathcal{M} \text {. }
$$

Proposition 3.1: For a PIN model, the SK capacity for a set of terminals $A \subseteq \mathcal{M}$, with $|A| \geq 2$, is

$$
C(A)=\min _{\lambda \in \Lambda(A)}\left[\sum_{1 \leq i<j \leq m}\left(\sum_{\substack{B \in \mathcal{B}(A): \\ i \in B, j \in B^{c}}} \lambda_{B}\right) I\left(X_{i j} \wedge X_{j i}\right)\right] .
$$

Remarks:

i) It is of interest in (3) that the SK capacity for a PIN model depends on the joint probability distribution of the underlying rvs only through a linear combination of the pairwise reciprocal mutual information terms.

ii) We note from [4, Th. 3] that additional independent randomization at the terminals in $\mathcal{M}$, enabled by giving them access to the mutually independent rvs $M_{1}, \ldots, M_{m}$, respectively, that are independent also of $\left(\tilde{X}_{1}^{n}, \ldots, \tilde{X}_{m}^{n}\right)$, does not serve to enhance SK capacity. Heuristically speaking, the mentioned independence of the randomization forces any additional "common randomness" among the terminals in $A$ to be acquired only through public communication, which is observed fully by the eavesdropper. On the other hand, randomization can serve to enhance secrecy generation for certain models (cf., e.g., [21])

Proof: The proof entails an application of the formula for SK capacity in [4] and [5] to the PIN model. For $B \in \mathcal{B}(A)$, denote $\tilde{X}_{B}=\left(\tilde{X}_{i}, i \in B\right)$. From ([5, Th. 3.1])

$$
C(A)=H\left(\tilde{X}_{1}, \ldots, \tilde{X}_{m}\right)-\max _{\lambda \in \Lambda(A)} \sum_{B \in \mathcal{B}(A)} \lambda_{B} H\left(\tilde{X}_{B} \mid \tilde{X}_{B^{c}}\right) .
$$

For the PIN model, since $\tilde{X}_{i}=\left(X_{i j}, j \in \mathcal{M} \backslash\{i\}\right)$, we observe in (4) that

$$
\begin{aligned}
H\left(\tilde{X}_{1}, \ldots, \tilde{X}_{m}\right) & =H\left(\left\{\left(X_{i j}, X_{j i}\right)\right\}_{1 \leq i<j \leq m}\right) \\
& =\sum_{1 \leq i<j \leq m} H\left(X_{i j}, X_{j i}\right)
\end{aligned}
$$

and

$$
\begin{aligned}
H\left(\tilde{X}_{B} \mid \tilde{X}_{B^{c}}\right)= & H\left(\tilde{X}_{\mathcal{M}}\right)-H\left(\tilde{X}_{B^{c}}\right) \\
= & \sum_{1 \leq i<j \leq m} H\left(X_{i j}, X_{j i}\right) \\
& -\sum_{\substack{1 \leq i<j \leq m, i \in B^{c}, j \in B^{c}}} H\left(X_{i j}, X_{j i}\right) \\
& -\sum_{\substack{i \in B^{c}, j \in B \\
=}} H\left(X_{i j}\right) \\
& \sum_{\substack{1 \leq i<j \leq m, i \in B, j \in B}} H\left(X_{i j}, X_{j i}\right) \\
& +\sum_{\substack{i \in B, j \in B^{c}\\
}} H\left(X_{i j} \mid X_{j i}\right) .
\end{aligned}
$$

A straightforward manipulation of (4), using (5), (6), gives

$$
\begin{aligned}
C(A)=\min _{\lambda \in \Lambda(A)} \sum_{1 \leq i<j \leq m} & {\left[H\left(X_{i j}, X_{j i}\right)\right.} \\
& -\left(\sum_{B \in \mathcal{B}(A):} \lambda_{B}\right) H\left(X_{i j}, X_{j i}\right) \\
& -\left(\sum_{\substack{B \in B, j \in B \\
i \in B, j \in B^{c}}} \lambda_{B}\right) H\left(X_{i j} \mid X_{j i}\right) \\
& \left.-\left(\sum_{\substack{B \in \mathcal{B}(A): \\
i \in B^{c}, j \in B}} \lambda_{B}\right) H\left(X_{j i} \mid X_{i j}\right)\right] .
\end{aligned}
$$

Since by (2)

$$
\sum_{\substack{B \in \mathcal{B}(A): \\ i \in B, j \in B}} \lambda_{B}=1-\sum_{\substack{B \in \mathcal{B}(A): \\ i \in B, j \in B^{c}}} \lambda_{B}=1-\sum_{\substack{B \in \mathcal{B}(A): \\ i \in B^{c}, j \in B}} \lambda_{B}
$$


we get

$C(A)$

$$
=\min _{\lambda \in \Lambda(A)}\left[\sum_{1 \leq i<j \leq m}\left(\sum_{\substack{B \in \mathcal{B}(A): \\
i \in B, j \in B^{c}}} \lambda_{B}\right)\left(\begin{array}{c}
H\left(X_{i j}, X_{j i}\right) \\
-H\left(X_{i j} \mid X_{j i}\right) \\
-H\left(X_{j i} \mid X_{i j}\right)
\end{array}\right)\right]
$$

thereby completing the proof.

An upper bound had been established for SK capacity for a general multiterminal source model [4, Example 4]. This bound was expressed in terms of the (Kullback-Leibler) divergence between the joint distribution of the rvs defining the underlying correlated sources and the product of the (marginal) distributions associated with appropriate partitions of these rvs, thereby measuring the minimum mutual dependence among the latter. The bound was particularized to the PIN model in [23], and is restated below in a slightly different form that will be used subsequently.

Let $\mathcal{P}$ be a partition of $\mathcal{M}=\{1, \ldots, m\}$, and denote the number of atoms of $\mathcal{P}$ by $|\mathcal{P}|$.

Lemma 3.2 [23]: The SK capacity $C(A), A \subseteq \mathcal{M}$, for the PIN model is bounded above according to

$$
\begin{gathered}
C(A) \leq \\
C^{u b}(A) \triangleq \min _{\mathcal{P}}\left(\frac{1}{|\mathcal{P}|-1}\right)\left[\sum_{\substack{1 \leq i<j \leq m \\
(i, j) \operatorname{crosses} \mathcal{P}}} I\left(X_{i j} \wedge X_{j i}\right)\right]
\end{gathered}
$$

where for a fixed $\mathcal{P}$, a pair of indices $(i, j)$ crosses $\mathcal{P}$ if $i$ and $j$ are in different atoms of $\mathcal{P}$. The minimization in the right side of (7) is over all partitions $\mathcal{P}$ of $\mathcal{M}$ for which every atom of $\mathcal{P}$ intersects $A$.

\section{B. SK Capacity and Steiner Tree Packing}

There exists a natural connection between SK generation for the PIN model and the combinatorial problem of tree packing in an associated multigraph.

Let $G=(V, E)$ be a multigraph, i.e., a connected undirected graph with no selfloops and with multiple edges possible between any vertex pair, whose vertex set $V=\mathcal{M}=\{1, \ldots, m\}$ and edge set $E=\left\{e_{i j} \geq 0,1 \leq i<j \leq m\right\}$, where $e_{i j}$ is the number of edges connecting the pair of vertices $i, j$, $1 \leq i<j \leq m$.

Definition 3: For $A \subseteq \mathcal{M}$, a Steiner tree of $G$ (for $A$ ) is a subgraph of $G$ that is a tree and whose vertex set contains $A$. A Steiner packing of $G$ is any collection of edge disjoint Steiner trees of $G$. Let $\mu(A, G)$ denote the maximum size of such a packing (cf. [11]).

We note that when $|A|=2$, a Steiner tree for $A$ always contains a path connecting the two vertices in $A$. Clearly, it suffices to take $\mu(A, G)$ to be the maximum number of edge disjoint paths connecting the two terminals in $A$.
Next, assume without any loss of generality in the PIN model that all pairwise reciprocal mutual information values $I\left(X_{i j} \wedge\right.$ $\left.X_{j i}\right), 1 \leq i \neq j \leq m$, are rational numbers. Let $\mathcal{N}$ denote the collection of positive integers $n$ such that the number of edges between any pair of vertices $i, j$ is equal to $n I\left(X_{i j} \wedge X_{j i}\right)$ is integer-valued for all $1 \leq i \neq j \leq m$; clearly, the elements of $\mathcal{N}$ form an arithmetic progression. For a PIN model, consider a sequence of associated multigraphs $\left\{G^{(n)}=\left(\mathcal{M}, E^{(n)}\right), n \in\right.$ $\mathcal{N}\}$, where $E^{(n)}, n \in \mathcal{N}$, is such that $e_{i j}=n I\left(X_{i j} \wedge X_{j i}\right)$. We term $\sup _{n \in \mathcal{N}} \frac{1}{n} \mu\left(A, G^{(n)}\right)$ as the maximum rate of Steiner tree packing in the multigraph $G=(\mathcal{M}, E)$. The connection between SK generation for the PIN model and Steiner tree packing is formalized here.

Theorem 3.3: For a PIN model,

i) the SK capacity satisfies

$$
C(A) \geq \sup _{n \in \mathcal{N}} \frac{1}{n} \mu\left(A, G^{(n)}\right)
$$

for every $A \subseteq \mathcal{M}$;

ii) when $|A|=2$, the SK capacity is

$$
\begin{aligned}
C(A) & =\sup _{n \in \mathcal{N}} \frac{1}{n} \mu\left(A, G^{(n)}\right) \\
& =C^{u b}(A) .
\end{aligned}
$$

\section{Remarks:}

i) The inequality in (8) can be strict, as shown by a specific example in a sequel paper [17]. See also the remark following Theorem 3.4 for a heuristic explanation.

ii) An exact determination of $\mu(A, G)$ is known to be NP-hard [3]. A nontrivial upper bound for $\mu(A, G)$, similar in form to (7), is known [12, para. 5, Sect. 1]. This bound can be extended to yield an upper bound for $\sup _{n \in \mathcal{N}} \frac{1}{n} \mu\left(A, G^{(n)}\right)$ which, in general, is inferior to that provided by $C(A)$ in (8).

Proof: i) The proof consists of two main steps. In the first step, fix an $\epsilon>0$ that is smaller than every positive $I\left(X_{i j} \wedge\right.$ $\left.X_{j i}\right), 1 \leq i<j \leq m$. Each pair of terminals $i, j$ with $I\left(X_{i j} \wedge X_{j i}\right)>0$, generates a (pairwise) SK $K_{i j}=K_{i j}^{(n)}$ of size $\left\lfloor n\left(I\left(X_{i j} \wedge X_{j i}\right)-\epsilon\right)\right\rfloor$ bits, using public communication $F_{i j}=F_{i j}^{(n)}$, and satisfying

$$
s\left(K_{i j} ; F_{i j}\right)=o_{n}(1)
$$

the existence of such an SK follows from [15]. The SK achievability scheme in [15] consists of a "weak" SK generated by Slepian-Wolf data compression, followed by "privacy amplification" to extract a "strong" SK. Note by the definition of the PIN model that $\left\{\left(K_{i j}, F_{i j}\right)\right\}_{1 \leq i<j \leq m}$ are mutually independent.

In the second step, consider the sequence of multigraphs $\left\{G_{\epsilon}^{(n)}=\left(\mathcal{M}, \widehat{E^{(n)}}\right)\right\}_{n=1}^{\infty}$, where $\widetilde{E^{(n)}}$ is such that the number of edges between any pair of vertices $i, j$ equals $\left\lfloor n\left(I\left(X_{i j} \wedge X_{j i}\right)-\epsilon\right)\right\rfloor$. We next show that every Steiner tree in a Steiner tree packing of $G_{\epsilon}^{(n)}$ yields one shared bit for the terminals in $A$ that is independent of the communication in that Steiner tree. Specifically, for edges $(i, j)$ and $\left(i, j^{\prime}\right), j \neq j^{\prime}$, with common vertex $i$ in the Steiner tree, vertex $i$ broadcasts to 
vertices $j, j^{\prime}$ the binary sum of two independent SK bits-one with $j$ and the other with $j^{\prime}$-obtained from the first step. This enables $i, j, j^{\prime}$ to share any one of these two bits, with the attribute that the shared bit is independent of the binary sum. This method of propagation ([4, Proof of Th. 5]) enables all the vertices in $A$, which are connected in the Steiner tree, to share one bit that is independent of all the broadcast binary sums from this tree. Therefore, the maximum number of such shared bits for the terminals in $A$ that can be generated by this procedure equals $\mu\left(A, G_{\epsilon}^{(n)}\right)$. Denote these shared bits (of size $\left.\mu\left(A, G_{\epsilon}^{(n)}\right)\right)$ and the communication messages generated by the mechanism in this second step by $K=K^{(n)}\left(\left\{K_{i j}\right\}_{1 \leq i<j \leq m}\right)$ and $F=F^{(n)}\left(\left\{K_{i j}\right\}_{1<i<j<m}\right)$, respectively.

We claim that $K$ constitutes an SK for $A$. Specifically, it remains to show that $K$ satisfies the secrecy condition (1) with respect to the overall communication in steps 1 and 2. To this end, we denote by $K_{R}^{(n)}\left(\left\{K_{i j}\right\}_{1 \leq i<j \leq m}\right)$ all the pairwise SK bits generated in the first step, that are residual from the maximal Steiner tree packing of $G_{\epsilon}^{(n)}$ used to generate $K$ by means of $F$. Clearly

$$
\left\{K_{i j}\right\}_{1 \leq i<j \leq m}=\left(K, F, K_{R}\right) .
$$

Moreover, since the total number of edges in any Steiner tree equals the sum of unity (i.e., the shared bit of $K$ ) and the number of bits of public communication for that shared bit, we have

$$
\left|\widetilde{E^{(n)}}\right|=\log |\mathcal{K}|+\log |\mathcal{F}|+\log \left|\mathcal{K}_{R}\right|
$$

where $\mathcal{K}, \mathcal{F}$ and $\mathcal{K}_{R}$ denote the respective ranges of $K, F$ and $K_{R}$. Note that $\log |\mathcal{K}|=\mu\left(A, G_{\epsilon}^{(n)}\right)$. Then

$$
\begin{aligned}
s( & \left.K\left\{F_{i j}\right\}_{1 \leq i<j \leq m}, F\right) \\
= & \log |\mathcal{K}|-H\left(K \mid\left\{F_{i j}\right\}_{1 \leq i<j \leq m}, F\right) \\
\leq & \log |\mathcal{K}|-H\left(K \mid\left\{F_{i j}\right\}_{1 \leq i<j \leq m}, F, K_{R}\right) \\
= & \log |\mathcal{K}|-H\left(K, F, K_{R} \mid\left\{F_{i j}\right\}_{1 \leq i<j \leq m}\right) \\
& +H\left(F, K_{R} \mid\left\{F_{i j}\right\}_{1 \leq i<j \leq m}\right) \\
= & \log |\mathcal{K}|-H\left(\left\{K_{i j}\right\}_{1 \leq i<j \leq m} \mid\left\{F_{i j}\right\}_{1 \leq i<j \leq m}\right) \\
& +H\left(F, K_{R} \mid\left\{F_{i j}\right\}_{1 \leq i<j \leq m}\right), \text { by }(11) \\
\leq & \log |\mathcal{K}|+s\left(\left\{K_{i j}\right\}_{1 \leq i<j \leq m} ;\left\{F_{i j}\right\}_{1 \leq i<j \leq m}\right) \\
& -\left|\widehat{E^{(n)}}\right|+H\left(F, K_{R}\right) \\
\leq & s\left(\left\{K_{i j}\right\}_{1 \leq i<j \leq m} ;\left\{F_{i j}\right\}_{1 \leq i<j \leq m}\right), \text { by }(12) \\
= & \sum_{1 \leq i<j \leq m} s\left(K_{i j} ; F_{i j}\right) \\
= & \frac{m(m-1)}{2} o_{n}(1)
\end{aligned}
$$

where the second-to-last equality is by the fact that $\left\{\left(K_{i j}, F_{i j}\right)\right\}_{1 \leq i<j \leq m}$ are mutually independent, and the last equality is by (10). The maximum rate of the SK thus generated is equal to $\lim _{n \rightarrow \infty} \frac{1}{n} \mu\left(A, G_{\epsilon}^{(n)}\right)$ which, since $\epsilon>0$ was arbitrary, equals $\sup _{n \in \mathcal{N}} \frac{1}{n} \mu\left(A, G^{(n)}\right)$.

ii) Suppose that $A=\{1,2\}$, and note from the paragraph after Definition 3 that $\mu(A, G)$ is the maximum number of edge disjoint paths in $G$ connecting terminals 1 and 2. It is clear that $\frac{1}{n} \mu\left(A, G^{(n)}\right)$ is nondecreasing in $n \in \mathcal{N}$, by the definition of
$G^{(n)}$. According to Menger's theorem [16], [2], given a multigraph $G=(\mathcal{M}, E)$, the maximum number of edge disjoint paths in $G$ connecting terminals 1 and 2 is equal to

$$
\left.\min _{\substack{\emptyset \neq B \subset \mathcal{M} \\ 1 \in B, 2 \in B^{c}}} \text { (number of edges that cross }\left\{B, B^{c}\right\}\right) .
$$

Applying this to $G^{(n)}$ as above, we have that for $n \in \mathcal{N}$

$$
\begin{aligned}
& \frac{1}{n} \mu\left(A, G^{(n)}\right) \\
& \quad=\frac{1}{n}\left[\min _{\substack{\emptyset \neq B \subset \mathcal{M} \\
1 \in B, 2 \in B^{c}}}\left(\sum_{\substack{1 \leq i<j \leq m: \\
(i, j) \text { crosses }\left\{B, B^{c}\right\}}} n I\left(X_{i j} \wedge X_{j i}\right)\right)\right] .
\end{aligned}
$$

It then follows that

$$
\begin{aligned}
C(A) & \geq \sup _{n \in \mathcal{N}} \frac{1}{n} \mu\left(A, G^{(n)}\right), \text { by (8) } \\
& =\min _{\substack{\emptyset \neq B \subset \mathcal{M} \\
1 \in B, 2 \in B^{c}}}\left(\sum_{\substack{1 \leq i<j \leq m: \\
(i, j) \operatorname{crosses}\left\{B, B^{c}\right\}}} n I\left(X_{i j} \wedge X_{j i}\right)\right) \\
& =C^{u b}(A), \text { by }(7) .
\end{aligned}
$$

The last equality follows upon noting that when $|A|=2$, the minimization in (7) is over only those partitions that contain two atoms, each of which includes terminal 1 and terminal 2, respectively. This proves (ii).

\section{SK Capacity and Spanning Tree Packing for $A=\mathcal{M}$}

When all the terminals in $\mathcal{M}$ seek a shared SK, i.e., when $A=\mathcal{M}$, a Steiner tree for $A$ is a spanning tree for $\mathcal{M}$. In this case, we show that the lower bound for SK capacity in Theorem 3.3 (i) is, in fact, tight. Specifically, we show that the algorithm in the proof of Theorem 3.3 yields an SK of maximum rate that coincides with the upper bound for $C(\mathcal{M})$ in Lemma 3.2.

Theorem 3.4: For a PIN model, the SK capacity $C(\mathcal{M})$ is

$$
\begin{aligned}
C(\mathcal{M}) & =\sup _{n \in \mathcal{N}} \frac{1}{n} \mu\left(\mathcal{M}, G^{(n)}\right) \\
& =C^{u b}(\mathcal{M}) .
\end{aligned}
$$

Remark: When $A \subset \mathcal{M}$, Steiner tree packing may not attain SK capacity. In SK generation, a helper terminal in $A^{c}$ helps link the user terminals in $A$ in complex ways through various combinations of subsets of $A$. In general, an optimal such linkage need not be attained by Steiner tree packing. However, when $|A|=2$, the two user terminals are either directly connected or are connected by a path through helpers in $A^{c}$; both can be accomplished by Steiner tree packing. When $A=\mathcal{M}$, the mentioned complexity of a helper is nonexistent.

Proof: The proof relies on a graph-theoretic result of NashWilliams [18] and Tutte [20], that gives a min max formula for the maximum size of spanning tree packing in a multigraph.

It is clear that $\frac{1}{n} \mu\left(\mathcal{M}, G^{(n)}\right)$ is nondecreasing in $n \in \mathcal{N}$, by the definition of $G^{(n)}$. By [18] and [20], given a multigraph 
$G=(\mathcal{M}, E)$, the maximum number of edge disjoint spanning trees that can be packed in $G$ is equal to

$$
\min _{\mathcal{P}}\left\lfloor\frac{1}{|\mathcal{P}|-1}(\text { number of edges that cross } \mathcal{P})\right\rfloor
$$

with the minimization being over all partitions $\mathcal{P}$ of $\mathcal{M}$. Applying this to $G^{(n)}$ as above, we have that for $n \in \mathcal{N}$

$$
\begin{aligned}
& \frac{1}{n} \mu\left(\mathcal{M}, G^{(n)}\right) \\
& \quad=\frac{1}{n}\left[\min _{\mathcal{P}}\left[\frac{1}{|\mathcal{P}|-1}\left(\sum_{\substack{1 \leq i<j \leq m: \\
(i, j) \text { crosses } \mathcal{P}}} n I\left(X_{i j} \wedge X_{j i}\right)\right)\right] .\right.
\end{aligned}
$$

Denoting by $D$ the quantity in [] above, it follows that

$$
\begin{aligned}
C(\mathcal{M}) & \geq \sup _{n \in \mathcal{N}} \frac{1}{n} \mu\left(\mathcal{M}, G^{(n)}\right), \text { by Theorem } 3.3 \\
& \geq \sup _{n \in \mathcal{N}}\left\{D-\frac{1}{n}\right\} \\
& \geq \min _{\mathcal{P}} \frac{1}{|\mathcal{P}|-1}\left(\sum_{\substack{1 \leq i<i \leq m: \\
(i, j) \text { crosses } \mathcal{P}}} I\left(X_{i j} \wedge X_{j i}\right)\right) \\
& =C^{u b}(\mathcal{M}), \text { by }(7) .
\end{aligned}
$$

The assertion in (13) is now immediate.

Last, the following observation is of independent interest. Given a combinatorial problem of finding the maximal packing of Steiner trees in a multigraph, we can always associate with it a problem of SK generation for an associated PIN model. By Theorem $3.3 \mathrm{i}$ ), the SK capacity for the PIN model yields an upper bound for the maximum rate of edge disjoint Steiner trees that can be packed in the multigraph; the upper bound is tight both in the case of path packing by Theorem 3.3 ii) and in the case of spanning tree packing by Theorem 3.4.

\section{DISCUSSION}

Our proofs of Theorems 3.3 and 3.4 give rise to explicit polynomial-time schemes for forming a group-wide SK for the terminals in $A$ from the collection of optimum and mutually independent SKs for pairs of terminals in $\mathcal{M}$ (namely the $K_{i j} s$ in the proof of Theorem 3.3). When $|A|=2$ or $A=\mathcal{M}$, our schemes achieve SK capacity. Specifically, the schemes combine known polynomial-time algorithms for finding a maximal collection of edge-disjoint paths (respectively, spanning trees) connecting the vertices in $A$ when $|A|=2$ (respectively, $A=\mathcal{M}$ ) [6]-[8] with the technique for SK propagation in each tree as in the proof of Theorem 3.3.

For a general multiterminal source model, the notions of wiretap secret key (WSK) [13], [1], [4] and private key (PK) [4] have also been proposed. Specifically, these notions involve an extra "wiretapped" terminal, say $m+1$, that observes $n$ i.i.d. repetitions of a rv $\tilde{X}_{m+1}$ with a given joint pmf with $\left(\tilde{X}_{1}, \ldots, \tilde{X}_{m}\right)$, and to which the eavesdropper has access. The key must now be concealed from the eavesdropper's observations of $\tilde{X}_{m+1}^{n}=\left(\tilde{X}_{m+1,1}, \ldots, \tilde{X}_{m+1, n}\right)$ and the public communication. The notion of a WSK requires that terminal $m+1$ not cooperate in key generation. The less restrictive notion of a PK allows cooperation by terminal $m+1$ by way of public communication. The corresponding capacities for the terminals in $A \subseteq \mathcal{M}$ are defined in the usual manner, and denoted by $C_{W}(A)$ and $C_{P}(A)$. We remark that in the context of a PIN model, terminal $m+1$ represents a compromised entity.

One model for the wiretapped rv $\tilde{X}_{m+1}$ entails its consisting of $\left(\begin{array}{c}m \\ 2\end{array}\right)$ mutually independent components, one corresponding to each pair $\left(X_{i j}, X_{j i}\right), 1 \leq i<j \leq m$, of legitimate correlated signals. This model is unresolved even in the simplest case of $m=2$ terminals [14], [1], [4], [9], [10]. Instead, we consider a different model which depicts the situation in which an erstwhile legitimate terminal $m+1$ becomes compromised. Specifically, the model now involves every legitimate terminal $i$ in $\mathcal{M}$ observing $n$ i.i.d. repetitions of the $\operatorname{rv}\left(\tilde{X}_{i}, X_{i, m+1}\right)$, while terminal $m+1$ observes $n$ i.i.d. repetitions of $\tilde{X}_{m+1}=\left(X_{m+1, j}, j \in \mathcal{M}\right)$. We argue in the following proposition that the WSK and PK capacities for this PIN model are the same as the SK capacity of a reduced PIN model obtained by disregarding terminal $m+1$ and with each legitimate terminal $i$ in $\mathcal{M}$ observing just $\tilde{X}_{i}^{n}$.

Proposition 4.1: It holds that

$$
C_{W}(A)=C_{P}(A)=C(A) .
$$

Proof: We shall prove that

$$
C(A) \stackrel{(a)}{\leq} C_{W}(A) \stackrel{(b)}{\leq} C_{P}(A) \stackrel{(c)}{\leq} C(A)
$$

The inequality $(b)$ is by definition. Next, let $K=K\left(\tilde{X}_{1}^{n}, \ldots, \tilde{X}_{m}^{n}\right)$ be a SK for $A$ achieved with communication $\mathbf{F}=\mathbf{F}\left(\tilde{X}_{1}^{n}, \ldots, \tilde{X}_{m}^{n}\right)$ for the reduced PIN model. Then $K$ is also a WSK since

$$
\begin{aligned}
s & \left(K ; \mathbf{F},\left(X_{m+1, j}^{n}, j \in \mathcal{M}\right)\right) \\
& =\log |K|-H\left(K \mid \mathbf{F},\left(X_{m+1, j}^{n}, j \in \mathcal{M}\right)\right) \\
& =s(K ; \mathbf{F})+I\left(K \wedge\left(X_{m+1, j}^{n}, j \in \mathcal{M}\right) \mid \mathbf{F}\right) \\
& =o_{n}(1)
\end{aligned}
$$

since $I\left(K, \mathbf{F} \wedge\left(X_{m+1, j}^{n}, j \in \mathcal{M}\right)\right)=0$, thereby establishing (a). In order to establish (c), we claim that every achievable PK rate is an achievable SK rate for the reduced PIN model upon using randomization at the terminals in $\mathcal{M}$; by Remark ii) after Proposition 3.1, (c) then follows. Since $\left(X_{m+1, j}^{n}, j \in \mathcal{M}\right)$ is independent of $\left(\tilde{X}_{1}^{n}, \ldots, \tilde{X}_{m}^{n}\right)$, any terminal in $\mathcal{M}$, say terminal 1 , can simulate $\left(X_{m+1, j}^{n}, j \in \mathcal{M}\right)$ and broadcast it to all the terminals. Next, each terminal $i$ in $\mathcal{M}$ can simulate $X_{i, m+1}^{n}$ conditioned on $\left(X_{m+1, j}^{n}, j \in\right.$ $\mathcal{M})=\left(x_{m+1, j}^{n}, j \in \mathcal{M}\right)$. This second step of randomization is feasible since $\left(\tilde{X}_{1}^{n}, \ldots, \tilde{X}_{m}^{n}\right), X_{1, m+1}^{n}, \ldots, X_{m, m+1}^{n}$ are conditionally mutually independent conditioned on $\left(X_{m+1, j}^{n}, j \in \mathcal{M}\right)=\left(x_{m+1, j}^{n}, j \in \mathcal{M}\right)$. Thus, each terminal $i$ in $\mathcal{M}$ now has access to $\left(\tilde{X}_{i}^{n}, X_{i, m+1}^{n}\right)$ while the eavesdropper observes $\left(X_{m+1, j}^{n}, j \in \mathcal{M}\right)$, so that the reduced PIN model for SK generation can be used to simulate a PIN 
model for PK generation with the given underlying joint pmf. Thus, any achievable rate of a PK for $A$ in the given PIN model for PK generation is an achievable rate of a PK for $A$ in the simulated model. Further, the latter PK is a fortiori an SK for $A$ in the reduced PIN model with randomization permitted at the terminals in $\mathcal{M}$. This establishes (c).

In the proof of achievability of SK capacity for the general multiterminal source model in [4], an SK of optimum rate was extracted from "omniscience," i.e., from a reconstruction by the terminals in $A$ of all the signals $\left(\tilde{X}_{i}^{n}, i \in \mathcal{M}\right)$ observed by the terminals in $\mathcal{M}$. In contrast, the scheme in Theorem 3.3 ii) (respectively, Theorem 3.4) for achieving SK capacity for a PIN model with $|A|=2$ (respectively $A=\mathcal{M}$ ) neither seeks nor attains omniscience; however, we note that omniscience can be attained by letting the terminals in $\mathcal{M}$ simply broadcast all the residual bits left over from a maximal path packing (respectively, maximal spanning tree packing).

We close with the observation that in the proof of Theorem 3.3 , the SK bit generated by each Steiner tree in Step 2 is exactly independent of the public communication in that tree. Thus, if the pairwise SKs in step 1 are "perfect" with zero security index, then so is the overall SK for $A$. It transpires that for the PIN model, there is a tight connection between "perfect secrecy generation" and "communication for perfect omniscience," redolent of the asymptotic connection in [4].

This new connection and the role of Steiner tree packing in attaining perfect omniscience and generating perfect secrecy are the subjects of a sequel paper [17].

\section{ACKNOWLEDGMENT}

The authors thank the anonymous referees for their helpful comments. P. Narayan thanks S. Khuller for the helpful pointer to [8].

\section{REFERENCES}

[1] R. Ahlswede and I. Csiszár, "Common randomness in information theory and cryptography, Part I: Secret sharing," IEEE Trans. Inf. Theory, vol. 39, pp. 1121-1132, Jul. 1993.

[2] A. Bondy and U. S. R. Murty, Graph Theory, ser. Graduate Texts in Mathematics. New York: Springer, 2008, vol. 244.

[3] J. Cheriyan and M. Salavatipour, "Hardness and approximation results for packing Steiner trees," Algorithmica, vol. 45, no. 1, pp. 21-43, 2006.

[4] I. Csiszár and P. Narayan, "Secrecy capacities for multiple terminals," IEEE Trans. Inf. Theory, vol. 50, pp. 3047-3061, Dec. 2004.

[5] I. Csiszár and P. Narayan, "Secrecy capacities for multiterminal channel models," Special Issue of IEEE Trans. Inf. Theory Inf. Theoret. Secur., vol. 54, pp. 2437-2452, Jun. 2008.

[6] E. A. Dinic, "An algorithm for the solution of the problem of maximal flow in a network with power estimation," Dokl. Akad. Nauk SSSR, vol. 194, pp. 754-757, 1970.

[7] J. Edmonds and R. M. Karp, "Theoretical improvements in algorithmic efficiency for network flow problems," in Combinatorial Structures and Their Applications. New York: Gordon and Breach, 1970, pp. 93-96.

[8] H. N. Gabow and H. H. Westermann, "Forests, frames, and games: Algorithms for matroid sums and applications," Algorithmica, vol. 7, pp. 465-497, 1992.
[9] A. Gohari and V. Anantharam, "Communication for omniscience by a neutral observer and information-theoretic key agreement of multiple terminals," in Proc. IEEE Int. Symp. Inf. Theory, Nice, France, Jun. 2007, pp. 2056-2060.

[10] A. Gohari and V. Anantharam, "New bounds on the information-theoretic key agreement of multiple terminals," in Proc. IEEE Int. Symp. Inf. Theory, Toronto, Ontario, Canada, Jul. 2008, pp. 742-746.

[11] M. Grötschel, A. Martin, and R. Weismantel, "Packing Steiner trees: A cutting plane algorithm and computational results," Math. Program., vol. 72, pp. 125-145, Feb. 1996.

[12] K. Jain, M. Mahdian, and M. R. Salavatipour, "Packing Steiner trees," in Proc. 14th ACM-SIAM Symp. Discr. Algorithms (SODA), Baltimore, MD, USA, Jan. 2003, pp. 266-274.

[13] U. M. Maurer, "Provably secure key distribution based on independent channels," in IEEE Workshop Inf. Theory, Eindhoven, The Netherlands, Jun. 1990.

[14] U. M. Maurer, "Secret key agreement by public discussion from common information," IEEE Trans. Inf. Theory, vol. 39, pp. 733-742, May 1993.

[15] U. M. Maurer, "The strong secret key rate of discrete random triples," in Communications and Cryptography: Two Sides of One Tapestry, R. E. Blahut, Ed. et al., Norwell, MA, 1994, pp. 271-285, 26.

[16] K. Menger, "Zur allgemeinen Kurventheorie," Fund. Math., vol. 10, pp. 96-115, 1927.

[17] S. Nitinawarat and P. Narayan, "Perfect omniscience, perfect secrecy, and Steiner tree packing," IEEE Trans. Inf Theory, vol. 56, no. 12, pp. 6490-6500, Dec. 2010.

[18] C. St. J. A. Nash-Williams, "Edge disjoint spanning trees of finite graphs," J. London Math. Soc., vol. 36, pp. 445-450, 1961.

[19] R. Renner and S. Wolf, "New bounds in secret-key agreement: The gap between formation and secrecy extraction," in Proc. EUROCRYPT 2003. New York: Springer-Verlag, 2003, vol. 2656, Lecture Notes in Computer Science, pp. 562-577.

[20] W. T. Tutte, "On the problem of decomposing a graph into $n$ connected factors," J. London Math. Soc., vol. 36, pp. 221-230, 1961.

[21] A. D. Wyner, "The wire-tap channel," Bell Syst. Tech. J., vol. 54, pp. 1355-1387, 1975.

[22] C. Ye, A. Reznik, and Y. Shah, "Extracting secrecy from jointly Gaussian random variables," in Proc. 2006 IEEE Int. Symp. Inf. Theory, Seattle, WA, Sep. 2006, pp. 2593-2597.

[23] C. Ye and A. Reznik, "Group secret key generation algorithms," in Proc. 2007 IEEE Int. Symp. Inf. Theory, Nice, France, Jun. 2007, pp. 2896-2900.

Sirin Nitinawarat (S'09) received the B.S.E.E. degree from Chulalongkorn University, Bangkok, Thailand, with first class honors in 2001, and the M.S.E.E. degree in 2003 from the University of Wisconsin, Madison.

He will receive the Ph.D. degree from the Department of Electrical and Computer Engineering and the Institute for Systems Research, University of Maryland, College Park, in December 2010. His research interests are in information theory and coding theory.

Chunxuan Ye (SM'10) received the B.Eng. (Hons) degree in electrical engineering from Shanghai Jiao Tong University, China, the M.Phil. degree in information engineering from the Chinese University of Hong Kong, and the Ph.D. degree in electrical and computer engineering from the University of Maryland, College Park, in 1997, 2000, and 2005, respectively.

He has been with InterDigital Communications, King of Prussia, PA, since 2005. His research interests are in the areas of information theory, communication theory, and wireless communication systems. These include information theoretic security, cooperative and relayed networks, network coding and source coding, wireless system prototyping platform, cognitive radio, and wireless communications networks. He has published more than 25 papers and book chapters. He has more than 15 pending U.S. patents. 
Alexander Barg (F'08) received the M.Sc. degree in applied mathematics and the $\mathrm{Ph} . \mathrm{D}$. degree in electrical engineering (information theory), the latter from the Institute for Information Transmission Problems (IPPI) Moscow, Russia, in 1987.

He has been a Senior Researcher with the IPPI since 1988. During 1995-1996, he was with the Technical University of Eindhoven, Eindhoven, the Netherlands. During 1997-2002, he was a Member of Technical Staff of Bell Labs, Lucent Technologies. Since 2003, he has been a Professor with the Department of Electrical and Computer Engineering and the Institute for Systems Research, University of Maryland, College Park. His research interests include coding and information theory and its links with computer science and mathematics.

Dr. Barg served as an Associate Editor for Coding Theory of the IEEE TRANSACTIONS ON INFORMATION THEORY during 1997-2000. He was the Technical Program Co-Chair of the 2006 IEEE International Symposium on Information Theory. He is presently a member of the Board of Governors of the Information Theory Society and serves on the Editorial Board of several journals including Problems of Information Transmission, SIAM Journal on Discrete Mathematics, and Advances of Mathematics in Communications.

Prakash Narayan (F'01) received the B.Tech. degree in electrical engineering from the Indian Institute of Technology, Madras, in 1976. He received the M.S. degree in systems science and mathematics in 1978 and the D.Sc. degree in electrical engineering in 1981, both from Washington University, St. Louis, MO.

$\mathrm{He}$ is Professor of Electrical and Computer Engineering with the University of Maryland, College Park, with a joint appointment at the Institute for Systems Research. He is also a founding member of the Center for Satellite and Hybrid Communication Networks, a NASA Commercial Space Center. He has held visiting appointments at ETH, Zurich; the Technion, Haifa; the Renyi Institute of the Hungarian Academy of Sciences, Budapest; the University of Bielefeld; the Institute of Biomedical Engineering (formerly LADSEB), Padova; and the Indian Institute of Science, Bangalore. His research interests are in multiuser information theory, communication theory, communication networks, cryptography, and information theory and statistics.

Dr. Narayan has served as an Associate Editor for Shannon Theory for the IEEE TRANSACTIONS ON INFORMATION THEORY; was Co-Organizer of the IEEE Workshop on Multi-User Information Theory and Systems, VA (1983); Technical Program Chair of the IEEE/IMS Workshop on Information Theory and Statistics, VA (1994); General Co-Chair of the IEEE International Symposium on Information Theory, Washington, DC (2001); and Technical Program Co-Chair of the IEEE Information Theory Workshop, Bangalore (2002). He currently serves as a Member of the Board of Governors of the IEEE Information Theory Society.
Alex Reznik (M'98) received the B.S.E.E. degree from the Cooper Union, New York, the S.M. degree in EECS from the Massachusetts Institute of Technology, Cambridge, and the $\mathrm{Ph} . \mathrm{D}$. degree in electrical engineering from Princeton University, Princeton, NJ, in 1996, 1998, and 2005, respectively.

During 2000-2002, he held a MURI fellowship at Princeton University. He has been with InterDigital, King of Prussia, PA, since 1999, where he is currently a Principal Engineer in the Advanced Communication Networks Group, leading a number of activities in the area of cognitive radio. His past contributions at InterDigital included technical leadership positions on projects in physical layer security, cellular modem architecture, and advanced receiver design. He holds a visiting faculty appointment with Winlab, Rutgers University, New Brunswick, NJ. His research interests are in information and communication theory and architecture and design of modern communication systems and devices. He is an inventor or coinventor on more than 40 granted U.S. patents

Dr. Reznik has been awarded several President's and CTO innovation awards at InterDigital. 